\title{
COVID-19 tsunami: the first case of a spinal cord injury patient in Italy
}

\author{
Gabriele Righi $^{1} \cdot$ Giulio Del Popolo ${ }^{1}$
}

Received: 28 March 2020 / Revised: 29 March 2020 / Accepted: 30 March 2020

(c) International Spinal Cord Society 2020

\begin{abstract}
Introduction We present the report of the first, to our best knowledge, case of COVID-19 in a tetraplegic person. Case presentation A 56-year-old male with AIS A C4 tetraplegia developed fever during the night, without any prodrome. His general practitioner suspected a urinary tract infection and prescribed him antibiotic therapy. After 2 days of antibiotic therapy the fever still persisted, so the individual was admitted to the local hospital and treated with broad-spectrum antibiotics. After 2 days he was transferred to our spinal unit. Considering the worsening of the chest X-ray and fever despite $48 \mathrm{~h}$ of broad-spectrum antibiotic therapy, we strongly suspected viral pneumonia. SARS-CoV-2 was detected and antiviral therapy with Lopinavir/Ritonavir, associated with hydroxychloroquine, was promptly started. Fever ceased after 2 days of therapy.

Discussion Blood test and chest X-ray findings in this patient were similar to previously published findings regarding COVID-19. One difference between this case and the known clinical course of COVID-19 is that did not develop cough. Another interesting feature of our case is that, despite tetraplegia, the clinical course was not severe. Persons with COVID-19 remain asymptomatic, these results underscore the need for rehabilitation and SCI professionals to have a high index of suspicion for COVID-19 in their inpatient and outpatient clients. Only inpatient with fever hase being tested for COVID-19. All new patients are submitted to SARS-COV-2 Test. Moreover, routine testing of patients who have to participate in therapy in common gym areas may be warranted.
\end{abstract}

\section{Introduction}

Coronavirus disease (COVID-19) is a respiratory tract infectious disease, first detected in Wuhan, China, and subsequently spread worldwide. It is caused by a novel virus, named severe acute respiratory syndrome coronavirus 2 (SARS-CoV-2) and has been recently declared a pandemic by the World Health Organization.

While most people with COVID-19 develop mild or uncomplicated illness, according to an epidemiological study conducted in China, $\sim 14 \%$ develop severe disease requiring hospitalization and oxygen support and 5\% require admission to an intensive care unit [1].

Risk factors for severe illness are not yet completely clear, although older patients and those with comorbid

Giulio Del Popolo

delpopolog@aou-careggi.toscana.it

Spinal Unit, Careggi University Hospital, Firenze, Italy medical conditions appear to be at higher risk for severe illness.

The aforementioned study found that the crude mortality rate for patients with no comorbidities was $\sim 0.9 \%$, while it was much higher for patients with comorbidities. The mortality rate among individuals with cardiovascular disease was $10.5 \%$, among individuals with diabetes was $7.3 \%$ and it was $6.3 \%, 6 \%$, and 5,6\%, respectively, among those individuals with chronic respiratory disease, hypertension, and cancer [1].

A recent meta-analysis of eight studies stated that, compared with individuals with mild disease, the pooled odds ratio of hypertension, respiratory system disease, and cardiovascular disease in severe patients were OR 2.36, 95\% CI: 1.46-3.83, OR 2.46, 95\% CI: 1.76-3.44, and OR 3.42 , 95\% CI: $1.88-6.22$, respectively [2].

Considering these data, it is extremely important to further investigate the effect of comorbid health conditions on the clinical course of this infectious disease. 


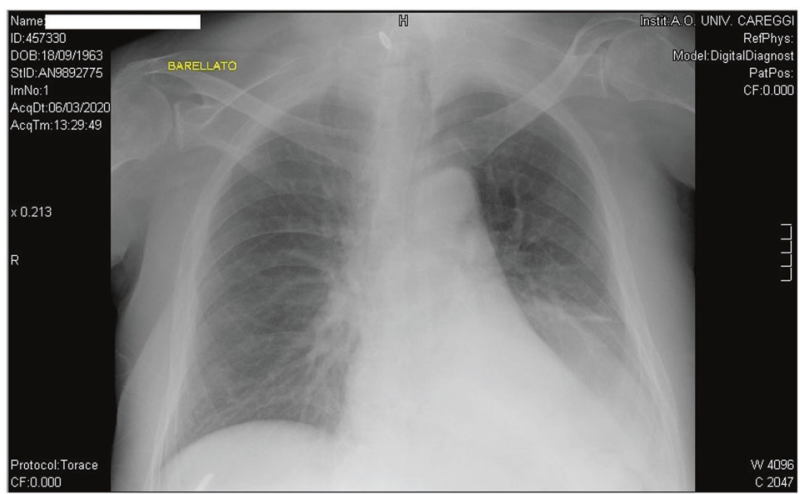

Fig. 1 Chest X-ray. Chest X-ray taken on admission to the short observation unit, showing perihilar congestion, interstitial opacity in lower right lobe and an opacity in the lower left lobe.

We therefore present the report of the first, to our best knowledge, case of COVID-19 in a person affected by tetraplegia.

\section{Case presentation}

G.G. is a 56 years old male affected by AIS A C4 tetraplegia since 2013, when he suffered a gun shot injury. He did not have any other relevant medical condition before his spinal cord injury (SCI). A former smoker, he ceased in 2010 (23 pack years). During the early phase of rehabilitation after his SCI he required a tracheostomy and was mechanically ventilated, but after weaning off mechanical ventilation, the tracheostomy tube was removed. He has a neurogenic bowel managed with permanent colostomy and neurogenic bladder managed with a suprapubic catheter and low-dose oxybutynin (5 mg daily). Current medications include rivaroxaban $20 \mathrm{mg}$ daily since 2014 due to recurrent deep vein thrombosis, gabapentin (400 $\mathrm{mg}$ twice a day) and tramadol (15 mg twice a day), both for neuropathic pain. He underwent plastic surgery in 2016 for reparation of a sacral pressure ulcer. In 2019 he was hospitalized three times for severe urinary tract infections, all sustained by gram negative bacteria. On his last hospital admission, in December 2019, he tested positive for colonization by carbapenemresistant Klebsiella pneumoniae on a rectal swab test.

At the beginning of March 2020 he developed fever during the night, without any prodrome. He had no other symptom and did not seek medical advice for an entire day. Since fever persisted, on the next day (around $36 \mathrm{~h}$ after fever had started) he contacted his general practitioner who prescribed him an antibiotic therapy targeted on the last urine culture available: trimethoprim/sulfamethoxazole 800/ $160 \mathrm{mg}$ twice daily.

After 2 days and a half of antibiotic therapy without any improvement, since his body temperature was still oscillating between 38 and $39{ }^{\circ} \mathrm{C}$, he was transported to the emergency department of our hospital and admitted to a short observation unit. Soon after the admission, samples for urine and blood cultures were collected, then chest $\mathrm{X}$-ray and abdominal ultrasound were performed. While the abdominal ultrasound examination showed no alteration, the chest X-ray showed signs of perihilar congestion, a worsening (compared to an X-ray performed in December 2019) of interstitial opacity in lower right lobe and an opacity in the lower left lobe-this latter opacity was reported as "unclear" because it was partially covered by elevated left hemidiaphragm (a chronic alteration in this patient) (Fig. 1).

Based on these findings the patient was treated with oral diuretics (Furosemide $25 \mathrm{mg}$ daily) and, since the most probable etiology of the fever was considered to be an urinary tract infection sustained by a bacterial strain resistant to trimethoprim/sulfamethoxazole, a broad-spectrum antibiotic therapy was started: meropenem $1 \mathrm{~g}$ three times a day and vancomycin $1 \mathrm{~g}$ twice a day.

Abnormalities of blood test on admission were limited to lymphocytopenia, mild hypokalemia, and slightly high level of C-reactive protein, while procalcitonin level showed no alterations.

The next day a worsening of leukopenia was found on routine blood tests: this finding was attributed to meropenem-induced leukopenia, so the patient was treated with filgrastim and meropenem was substituted with amikacin.

After 2 days of stay in the short observation unit the patient was transferred to our spinal unit, into a private room.

When asked about his symptoms he reported just fever and mild dyspnea, and denied cough, rhinorrhea, sore throat and any other symptom related to upper respiratory tract involvement.

Fever was still present (it was 6th day since the beginning of symptoms) despite 4 days and a half of antibiotic therapy-the last two with broad-spectrum antibiotics.

Physical examination performed on admission to our ward found no abdominal alterations, while respiratory examination revealed diffusely decreased breath sounds (a chronic finding) and the presence of rales in both lower lobes.

Manually assisted cough was performed in order to try to reduce dyspnea, but it produced only a small amount of secretions.

On admission we performed arterial blood gas test on room air and found mild hypoxia and respiratory alkalosis with metabolic compensation-the results were the following: $\mathrm{pH} 7.44 \mathrm{pO}_{2} 58.2 \mathrm{mmHg}, \mathrm{pCO}_{2} 29.4 \mathrm{mmHg}, \mathrm{sO}_{2}$ $94.3 \%, \mathrm{CHCO}_{3}{ }^{-} 22 \mathrm{mmol} / \mathrm{L}, \quad \mathrm{cLAC} 1 \mathrm{mmol} / \mathrm{L}, \quad \mathrm{ABE}$ $2.9 \mathrm{mmol} / \mathrm{L}, \mathrm{SBE}-3.9 \mathrm{mmol} / \mathrm{L}$. 


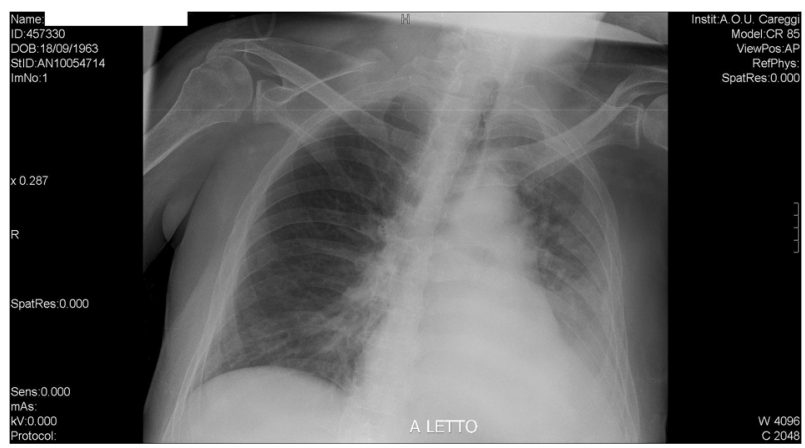

Fig. 2 Chest X-ray. This examination was perfomed after the transfer to the spinal unit (2 days and a half after the previous one) showing a worsening of the opacity in the lower left lobe.

We therefore resumed low-dose oxygen therapy $(1 \mathrm{~L} / \mathrm{min}$ during daytime, $2 \mathrm{~L} / \mathrm{min}$ at night, via face mask) which had been administered also during the previous days.

On the first day in the SCI ward we repeated the chest $\mathrm{X}$-ray, which was read as worsening of the consolidation in the lower left lobe and the persistence of both perihilar congestion and interstitial opacity in lower right lobe (Fig. 2)

Considering the worsening of the chest X-ray and persistence of fever despite $48 \mathrm{~h}$ of broad-spectrum antibiotic therapy, we strongly suspected a viral pneumonia and requested a consultation to an infectious disease specialist.

At that time, our hospital's procedures required the opinion of an infectious disease specialists before requesting a nasopharyngeal swab for the detection of SARS-CoV-2.

Following his advice, we substituted amikacin with piperacillin/tazobactam $(4 / 0.5 \mathrm{~g}$ three times a day) repeated blood culture and performed nasopharyngeal swabs for the detection of Influenza A virus, Influenza B virus and SARSCoV-2, using reverse transcriptase-polymerase chain reaction (RT-PCR) assays.

The antibiotic therapy was modified in order to reduce potential nephrotoxicity.

While we were waiting for the results of the nasopharyngeal swabs, we were notified the negativity of the urine culture taken during the stay in the short observation unit.

After SARS-CoV-2 was detected, the patient was transferred to the infectious disease unit of our hospital and antiviral therapy with Lopinavir/Ritonavir (400/100 mg twice a day) associated with hydroxychloroquine $(200 \mathrm{mg}$ twice a day) was promptly started.

Vancomycin therapy was suspended while piperacillin/ tazobactam was continued to guarantee coverage against bacterial infections.

Fever ceased after two days of therapy (10 days after the beginning of symptoms) and low-dose oxygen therapy was no longer required three days after transfer to the infectious disease unit.
Antiviral therapy, hydroxychloroquine and piperacillin/ tazobactam were stopped after one week of stay in the infectious disease unit, when the patient was discharged and sent home.

Currently, the criteria used in our hospital to discharge COVID-19 patients are based on a recent technical report by the European Centre for Disease Prevention and Control: (1) absence of fever for more than 3 days (2) improved respiratory function, with no need to administer oxygen (3) clinician assessment.

Arterial blood gas test performed on room air prior to discharge showed the following results: $\mathrm{pH} 7.4, \mathrm{pO}_{2}$ $74.9 \mathrm{mmHg}, \quad \mathrm{pCO}_{2} 33.8 \mathrm{mmHg}, \quad \mathrm{sO}_{2} 94.2 \%, \mathrm{HCO}_{3}{ }^{-}$ $22 \mathrm{mmol} / \mathrm{L}$.

The blood tests the day before discharge were normal, except for slightly high C-reactive protein levels $(10 \mathrm{mg} / \mathrm{L})$.

The day prior to discharge a nasopharyngeal swab for the detection of SARS-CoV-2 was repeated and resulted positive, so both the patient and his caregiver were put on home isolation and provide surgical face masks and gloves.

Since bladder and bowel management is eased by suprapubic catheter and colostomy, it was possible for a single caregiver to completely assist him.

\section{Discussion}

COVID-19 is a novel disease and thus we, using the words of $\operatorname{Dr}$ Anthony Fauci, are currently "navigating the uncharted" [3].

Nonetheless, there is an increasing amount evidence regarding this infectious disease. A large retrospective cohort study held in Wuhan, China, found that lymphocytopenia was the most common blood test alteration detected in COVID-19 patients [4]. The same study found also that age and lymphopenia along with leukocytosis, elevated ALT, lactate dehydrogenase, high-sensitivity cardiac troponin I, creatine kinase, d-dimer, serum ferritin, IL-6, prothrombin time, creatinine, and procalcitonin were also associated with in-hospital death. Another finding of that study is that mortality is particularly higher in patients with diabetes or coronary heart disease; this finding is consistent with the results of the large epidemiologic studies cited in the introduction of the present paper [1,2].

Regarding chest X-ray, the findings in our patient are consistent with literature, since bilateral pulmonary infiltration is the most common X-ray alteration detected in COVID-19 patients $[1,4,5]$.

An important difference between our case and the usual clinical course of COVID-19 is that our patient never developed dry cough, the second most common symptom of COVID-19 after fever [4, 6]. This should not be surprising, because it is well known that persons affected by tetraplegia 
have limited ability to cough due to paralysis of expiratory muscles, i.e., abdominal and internal intercostal muscles $[7,8]$

This case emphasizes, for physicians and health care professions with little or no experience in treating individuals with SCI that the absence of cough in an individual with tetraplegia patient does not rule out the diagnosis of viral pneumonia. Moreover, as some patients who are COVID-19 positive do not have symptoms one needs to have a high index of suspicion for COVID-19 in all individuals with SCI and fever, regardless of their symptoms.

Regarding caregivers, they should be also particularly observant to respect advices about social restriction, as SCI patients could develop a underhand form of disease, difficult to diagnose.

Another interesting feature of our case is that, despite tetraplegia was a major comorbidity, the clinical course was not severe, with a complete healing in little more than 2 weeks and no need for mechanical ventilation: this is in contrast with the acknowledged SCI induced immune depression syndrome [9]

It is also known that after SCI innate immunity (which has a key role in the immune response to a novel virus) is altered [10] and, in an animal model, it was demonstrated that the impaired immune responses following SCI lead to reduced ability to control viral infection [11].

In our opinion this discrepancy between an expected severe disease and the actual mild-to-moderate clinical picture could be explained by both the relatively young age of the subject and the absence of other comorbidities (diabetes, cardiovascular diseases, hypertension, chronic obstructive pulmonary disease).

Another possible explanation for the mild clinical course of COVID-19 in this patient may be a postulated different susceptibility to SARS-CoV-2 based on genetic [12] and epigenetic [13] factors and the actual viral load that he received upon infection.

Our last remark is that, since COVID-19 has extremely rapid rate of transmission $[1,14]$ it should be suspected in tetraplegic and paraplegic patients with fever even if it is not widespread in their place of residence: when our patient developed fever, only a single case of COVID-19 had been detected in the 200,000 inhabitants city in central Italy where he is living.

As for our spinal unit, thanks to accurate preventive measures no other inpatient was infected and they were able to continue their rehabilitation program. We have ready a special section dedicated to SCI patients with COVID-19. Every new patient has to undergo a chest X-ray and to be tested with a nasopharyngeal swab to detect SARS-CoV-2 before being admitted to our ward-after taking the nasopharyngeal swab, the patient is transferred to a private room and protected with face mask and gloves to be sure that he does not get infected in the time between the test and the transfer to our ward. Visits from friends and relatives have temporally been suspended, they communicate by phone or tablet. Our outpatient clinic activities have been limited to urgent cases only, while standard and follow-up visits are performed on video or phone call and by e-mail to transfer documents and prescription.

Only inpatient with fever have being tested for SARSCoV-2 and none, with the exception of the subject of this case report, has tested positive yet. But day-by-day we need to introduce new restrictions.

Finally, we, together with other spinal unit from all over Italy, are currently creating a national database of SCI individuals affected by COVID-19.

\section{Compliance with ethical standards}

Conflict of interest The authors declare that they have no conflict of interest.

Publisher's note Springer Nature remains neutral with regard to jurisdictional claims in published maps and institutional affiliations.

\section{References}

1. Novel Coronavirus Pneumonia Emergency Response Epidemiology Team (TNCEPERE) The epidemiological characteristics of an outbreak of 2019 novel coronavirus diseases (COVID-19) in China. Zhonghua Liu Xing Bing Xue Za Zhi. 2020;41:145-51.

2. Yang J, Zheng Y, Gou X, Pu K, Chen Z, Guo Q, et al. Prevalence of comorbidities in the novel Wuhan coronavirus (COVID-19) infection: a systematic review and meta-analysis. Int J Infect Dis. 2020. https://doi.org/10.1016/j.ijid.2020.03.017.

3. Fauci AS, Lane HC, Redfield RR. Covid-19-navigating the uncharted. N Engl J Med. 2020. https://doi.org/10.1056/ NEJMe200238.

4. Zhou F, Yu T, Du R, Fan G, Liu Y, Liu Z, et al. Clinical course and risk factors for mortality of adult inpatients with COVID-19 in Wuhan, China: a retrospective cohort study. Lancet. 2020;395: 1054-1062.

5. Zu ZY, Jiang MD, Xu PP, Chen W, Ni QQ, Lu GM, et al. Coronavirus disease 2019 (COVID-19): a perspective from China. Radiology. 2020. https://doi.org/10.1148/radiol.2020200490. [Epub ahead of print].

6. Huang C, Wang Y, Li X, Ren L, Zhao J, Hu Y, et al. Clinical features of patients infected with 2019 novel coronavirus in Wuhan, China. Lancet. 2020;395:497-506.

7. Fogarty MJ, Sieck GC. Spinal cord injury and diaphragm neuromotor control. Exp Rev Respir Med. 2020. https://doi.org/10. 1080/17476348.2020.1732822. [E-pub ahead of print].

8. Taylor PN, Tromans AM, Harris KR, Swain ID. Electrical stimulation of abdominal muscles for control of blood pressure and augmentation of cough in a C3/4 level tetraplegic. Spinal Cord. 2002;40:34-36.

9. Meisel C, Schwab JM, Prass K, Meisel A, Dirnagl U. Central nervous system injury-induced immune deficiency syndrome. Nat Rev Neurosci. 2005;6:775-86. 
10. Campagnolo DI, Dixon D, Schwartz J, Bartlett JA, Keller SE. Altered innate immunity following spinal cord injury. Spinal Cord. 2008;46:477-81.

11. Held KS, Steward O, Blanc C, Lane TE. Impaired immune responses following spinal cord injury lead to reduced ability to control viral infection. Exp Neurol. 2010;226:242-53.

12. Cai G, Cui X, Zhu X, Zhou J. A hint on the COVID-19 risk: population disparities in gene expression of three receptors of SARS-CoV. Preprints. 2020;2020020408. https://doi.org/10.20944/ preprints202002.0408.v1.
13. Corley, MJ, Ndhlovu, LC. DNA methylation analysis of the COVID-19 host cell receptor, angiotensin I converting enzyme 2 gene (ACE2) in the respiratory system reveal age and gender differences. Preprints. 2020;2020030295. https://doi.org/10.20944/ preprints202003.0295.v1.

14. Kucharski, AJ, Russell, TW, Diamond, C, Liu, Y, Edmunds, J, Funk, S, et al. Early dynamics of transmission and control of COVID-19: a mathematical modelling study. Lancet Infect Dis. 2020. https://doi.org/10.1016/S1473-3099(20)30144-4. [E-pub ahead of print]. 